male). Their mean age was $70.63 \pm 10.60$ years (range, $46-96$ years). The description of the causes of fracture, its level, the access route and the cement leaks are expressed in TABLE. The median time from fracture to vertebroplasty was 3 months [1-6]. $78 \%$ of the fractures were osteoporotic and had a better response to pain at 6 months than all other fractures $(p<0.05)$, although at 12 months there were no differences $(p=0.42)$. Only $12.5 \%$ had refracture. Pain control and vertebral refracture did not differ between neither the access rout nor the cement leakage and its magnitude. In the 12-month follow-up, 7 patients died (6 due to neoplasia, 1 due to sudden death) and no case was related to vertebroplasty.

Conclusions: In our experience vertebroplasty has been shown to be an effective technique for the control of refractory pain in vertebral fractures regardless of cause and time of evolution. Likewise, only $12.5 \%$ presented refracture in the following 12 months to the procedure. According to our study we can consider vertebroplasty as an effective and safe alternative in this type of patients.

Disclosure of Interest: None declared

DOI: 10.1136/annrheumdis-2017-eular.3543

\section{SAT0601 TREATMENT OF CARPAL TUNNEL SYNDROME (CTS) WITH ESWT: A SHAM CONTROLLED DOUBLE BLINDED RANDOMISED STUDY}

Ö. Karataş ${ }^{1}$, S. Catal ${ }^{2}$, E.A. Gökmen ${ }^{3}$, N. Samanci ${ }^{4} \cdot{ }^{1}$ Physical Medicine and Rehabilitation, Korkuteli Public Hospital; ${ }^{2}$ Physical Medicine and Rehabilitation, Akdeniz University School of Medicine; ${ }^{3}$ Physical Medicine and Rehabilitation, Antalya Training and Research Hospita; ${ }^{4}$ Physical Medicine and Rehabilitation, Akdeniz University School of Medicine, Antalya, Turkey

Background: The carpal tunnel syndrome (CTS) is the most common neuropathy (1). The etiology isn't properly known, but CTS is produced by the chronic compression of median nerve while passing through carpal canal can be associated with this disease (2).

Objectives: The aim of this study was to investigate the efficiency of extracorporeal shockwave therapy (ESWT) in the treatment of CTS.

Methods: 49 hand with the diagnosis of CTS were included in the study. Patients were randomised in ESWT ( $n=29$ hands) and sham ( $n=20$ hands) groups. Patients were randomly allocated to receive 1 session per week for 3 weeks of either sham or active ESWT. All patients were prescribed with tendon and nerve gliding exercises and hand-wrist splint which used night. Patients were evaluated before the treatment, and at the end of the first week, first month and third month after the last ESWT treatment session with Boston Scale (symptom severity and functional capacity), Visual Analogue Scale (VAS) for pain and paresthesia assessment, for muscle strength hand gross grasp and electroneurophysiological parameters.

Results: A total of 38 patients completed the study with 29 wrists in active ESWT and 20 wrists in sham ESWT groups. Groups were similar in age, sex, duration of symptoms, hand gross grasp and electrodiagnostic parameters $(P>0.05)$. In both groups, significant improvements were observed in VAS, Boston Scale and hand gross grasp after treatment. In both groups, there was no statistically significant improvement in electromyographic variables after treatment $(p>0.05)$. However there was no significiant differrence between two groups in all of clinical and electrodiagnostic parameters.

Conclusions: Although ESWT was effective in symptoms in CTS but this efficacy isn't superior to placebo. Our results indicated that ESWT was effective in pain and clinical variables in CTS. Wider and high-quality studies are needed to further demonstrate the effectiveness of ESWT in treatment of CTS.

References:

[1] Ozturk K, Esenyel CZ, Sonmez M, et al: Comparison of carpal tunnel injection techniques: A cadaver study. Scand J Plast Reconstr Surg Hand Surg 2008:42:300-4.

[2] Gelberman RH, Hergenroeder PT, Hargens AR, Lunborg GN, Akeson WH. The carpal tunnel syndrome. A study of carpal canal pressures. J Bone Joint Surg Am 1981;63:380-3.

Disclosure of Interest: None declared

DOI: 10.1136/annrheumdis-2017-eular.5363

\section{SAT0602 MANAGEMENT OF EPICONDYLITIS WITH SINGLE LOCAL INJECTION OF SODIUM HYALURONATE}

\section{R. Petrella, A. Cogliano, A. Decruze. Western University, London, Canada}

Background: Lateral elbow epicondylitis, also known as tennis elbow, is a common musculoskeletal condition causing pain and functional impairment in daily activities. ${ }^{1}$ It affects between $40-50 \%$ of recreational tennis players at some time. ${ }^{2}$ Sodium Hyaluronate $(\mathrm{SH})$ is a natural biological substance which has proven to be effective to improve pain and function in osteoarthritic patients with low incidence of side effects. ${ }^{3}$ Similarly, the administration of periarticular injections of $\mathrm{SH}$ can be an alternative approach to treat chronic epicondylitis. ${ }^{4}$ Objectives: To evaluate the efficacy and safety of a single periarticular injection with $\mathrm{SH}$ in the treatment of epicondylitis.

Methods: A single-site, and placebo-controlled trial was conducted in patients with chronic epicondylitis. Patients' condition was assessed at baseline and afterwards they were randomized 1:1 to receive a single $2.5 \mathrm{ml}$ injection of $\mathrm{SH}$ (manufactured by Tedec Meiji Farma SA) or placebo (saline) at the point of maximal pain at the lateral epicondyle. Additionally, standard of care (RICE: Rest, Ice, Compression and Elevation) was prescribed to both groups. Efficacy assessments were done at days 30 and 90 and included VAS $(0-10 \mathrm{~cm})$ pain at rest and assessment of grip strength, patient global satisfaction, patient assessment of normal function and physician global assessment of elbow injury (all measured using 5-point categorical scale). Adverse events were recorded for safety purposes.

Results: A total of 60 patients were included and completed the study procedures. Both groups were homogeneous at baseline. A statistically significant reduction from baseline in VAS pain at rest and after grip testing was observed at 30 and 90 days in both treatment groups $(p<0.05)$. Besides, inter-group comparison showed statistically significant differences in favour of $\mathrm{SH}$ group at 30 and 90 days $(p<0.05)$. This was associated with significantly greater grip strength, patient global satisfaction and assessment of normal elbow function in SH group vs placebo $(p<0.05)$. Improvement of elbow injury assessed by the physician was also statistically greater in patients treated with $\mathrm{SH}$ compared to placebo $(p<0.05)$. No adverse events were recorded.

Conclusions: A single local injection of $\mathrm{SH}$ administered to patients with epicondylitis was significantly superior to placebo improving pain at rest and after grip testing, through all the study follow up period. The treatment was highly satisfactory for both physicians and patients and there were no safety concerns.

\section{References:}

[1] Chourasia AO, Buhr KA, Rabago DP, Kijowski R, Lee KS, Ryan MP, GrettieBelling JM, Sesto ME. Relationships between biomechanics, tendon pathology, and function in individuals with lateral epicondylosis. J Orthop Sports Phys Ther. 2013 Jun;43(6):368-78. doi:. 10.2519/jospt.2013.4411.

[2] Taylor SA, Hannafin JA. Evaluation and management of elbow tendinopathy. Sports Health 2012; 4(5): 384-93.

[3] Bannuru RR, Schmid CH, Kent DM, Vaysbrot EE, Wong JB, McAlindon TE. Comparative Effectiveness of Pharmacologic Interventions for Knee Osteoarthritis: A Systematic Review and Network Meta-analysis. Ann Intern Med 2015; 162(1): 46-54.

[4] Petrella RJ, Cogliano A, Decaria J, Mohamed N, Lee R. Management of tennis elbow with sodium hyaluronate periarticular injections. Sports Med Arthrosc Rehabil Ther Technol 2010; 2:4.

Disclosure of Interest: None declared

DOI: 10.1136/annrheumdis-2017-eular.2986

\section{SAT0603 AN INNOVATIVE TREATMENT MODALITY FOR ACUTE ILIO-TIBIAL BAND SYNDROME IN RUNNERS: LOCAL HYALURONATE + BOTULINUM TOXIN IN A PROSPECTIVE COHORT OF 45 ATHLETES}

\section{R. Petrella, A. Decruze, J. Decaria. Western University, London, Canada}

Background: lliotibial band syndrome (ITBS) is the most common cause of lateral knee pain in runners ${ }^{1}$. It is an overuse injury that results from repetitive friction of the iliotibial band (ITB) over the lateral femoral epicondyle. Initial treatment includes activity modification, nonsteroidal anti-inflammatory medication, taping, stretching exercise and in severe cases, a corticosteroid injection ${ }^{2}$. Treatment of symptoms and return to activity are variable and can be intractable.

Objectives: To evaluate the efficacy and safety on pain and return to activity of a single, local injection of ITBS with combination hyaluronate and Botulinum toxin in 45 runners.

Methods: 45 runners with at least grade 2 ITBS underwent baseline investigations including pain following symptom-limited treadmill running test during which pain was recorded on a visual analogue scale (VAS 0-10) every minute. Runners then had injection in the area where the iliotibial band crosses the lateral femoral condyle with $2.5 \mathrm{ml}$ combination hyaluronate $(750-1300 \mathrm{kDa})$ with $40 \mathrm{U}$ Botulinum toxin. Additionally, standard of care (RICE: Rest, Ice, Compression and Elevation) and stretching was prescribed but participants were instructed not to use NSAIDS or taping. The same pain VAS measures as well as peak exercise time, patient global satisfaction and patient assessment of normal running function (all measured using 5-point categorical scale) were repeated after 2, 7, 14 and 30 days. The primary outcome was peak pain during symptom-limited treadmill running.

Adverse events were recorded for safety purposes.

Results: 45 consecutive runners with acute (within 7 days) ITBS were included and completed the study procedures. A statistically significant reduction from baseline in VAS peak treadmill exercise was observed at all time points $(p<0.05)$. This was associated with significantly longer exercise time at 7, 14 and 30 days. Patient global satisfaction was increased progressively after 7, 14 and 30 days and assessment of normal running function was described in $>75 \%$ at 14 days. No serious adverse were reported. 3 subjects described transient ( $<24$ hours) weakness in knee extension and 2 subjects described mild pain at the time and location of injection.

Conclusions: A single local injection of combination hyaluronate + Botulinum toxin for ITBS in runners improved pain and exercise time with treadmill running by 7 days post treatment and continued to 30 days. This treatment was satisfactory to runners and resulted in few, limited adverse events.

\section{References:}

[1] Louw M, Deary C. The biomechanical variables involved in the aetiology of iliotibial band syndrome in distance runners - A systematic review of the literature. Phys Ther Sport. 2014 Feb;15(1):64-75. 
[2] Fredericson $M$, Wolf $C$. lliotibial band syndrome in runners: innovations in treatment.Sports Med. 2005;35(5):451-9.

Disclosure of Interest: None declared

DOI: 10.1136/annrheumdis-2017-eular.3552

\section{SAT0604 AUTOLOGOUS BLOOD AND CORTICOSTEROID LOCAL INJECTION IN TREATMENT OF PLANTAR FASCIITIS (RANDOMIZED, CONTROLLED MULTICENTER CLINICAL TRIAL)}

A. Karimzadeh, S.A. Raeissadat, S. Erfani fam, L. Sedighipour. Physical Medicine \& Rehabilitation Research Center, Tehran, Iran, Islamic Republic Of

Background: Plantar fasciitis is the most common cause of heel pain. Local injection modalities are among treatment options in patients with resistant pain.

Objectives: The aim of the present study was to evaluate the effect of local autologous blood compared with corticosteroid local injection in treatment of plantar fasciitis.

Methods: In this randomized controlled multicenter study, 36 patients with chronic plantar fasciitis were recruited. Patients were allocated randomly into 3 treatment groups: local autologous blood, local corticosteroid injection and control groups receiving no injection. Patients were assessed with Visual Analogue Scale (VAS), Pressure Pain Threshold (PPT) and Plantar Fasciitis Pain/Disability Scale (PFPS) before treatment, 4 and 12 weeks post therapy.

Results: Variables of pain and function improved significantly in both corticosteroid and autologous blood groups compared to control group. At 4 weeks following treatment, patients in corticosteroid group had significantly lower levels of pain than patients in autologous blood and control groups (higher PPT level, lower PFPS and VAS). After 12 weeks of treatment both corticosteroid and autologous blood groups had lower average levels of pain than control group. The corticosteroid group showed an early sharp and then more gradual improvement in pain scores but autologous blood group had steady gradual drop in pain.

Conclusions: Autologous blood and corticosteroid local injection both can be considered as effective methods in the treatment of chronic plantar fasciitis. These treatments decrease pain and improve function significantly compared to control group.

Disclosure of Interest: None declared

DOI: 10.1136/annrheumdis-2017-eular.1771

\section{SAT0605 LOCAL PROGESTERONE INJECTION: NEW OPTION FOR MANAGEMENT OF CARPAL TUNNEL SYNDROME}

S.A. Raeissadat ${ }^{1}$, L. Sedighipour ${ }^{1}$, S. Shahraeeni ${ }^{2}$. ${ }^{1}$ Physical Medicine \& Rehabilitation Research Center; ${ }^{2}$ Physical medicine and rehabilitation research center, Tehran, Iran, Islamic Republic Of

Background: A number of studies, have demonstrated the neuroprotective effects of progesterone and its influence on the recovery after neural injury. Few studies investigated the efficacy of local progesterone in Carpal Tunnel Syndrome.

Objectives: The objective of this study was to compare the long term effects of progesterone versus corticosteroid local injections in patients with mild and moderate carpal tunnel syndrome.

Methods: In this randomized clinical trial, 78 hands with Carpal Tunnel Syndrome were assigned to two groups. Patients were treated with a single local injection of triamcinolone acetonide in one group and single local injection of hydroxy progesterone in the other group. Variables including pain (based on Visual Analogue Scale), symptom severity and functional status (based on Bostone/Levine symptom severity and functional status scale) and nerve conduction study were evaluated before and 6 months after the treatments.

Results: All outcome measures including pain, functional scales and electrophysiologic findings improved in both corticosteroid and progesterone groups and there were no meaningful differences between two groups regarding mentioned variables. However, functional outcome was significantly better in progesterone compared to corticosteroid group at 6 month follow up $(\mathrm{P}=0.04)$

Conclusions: This study demonstrated the efficacy of progesterone local injection in mild and moderate CTS at long term follow up. Furthermore, local progesterone can be superior to corticosteroid injection for relieving symptoms and improving functional and electrophysiologic findings at long term follow up.

Disclosure of Interest: None declared

DOI: 10.1136/annrheumdis-2017-eular.1774

\section{SAT0606 IS THE LEVEL OF PHYSICAL ACTIVITY AN IMPORTANT FACTOR FOR LOW BACK PAIN AMONG STUDENTS OF UNIVERSITY?}

V. Akduman ${ }^{1}$, E. Mete ${ }^{1}$, I. Demirbuken ${ }^{1}$, G. Ogren ${ }^{2} .{ }^{1}$ Physiotherapy and Rehabilitation; ${ }^{2}$ Nutrition and Dietetic, Marmara University, Istanbul, Turkey

Background: Back pain is a major global health problem, causing greater disability worldwide than any other condition. Regular physical activity is widely believed to have important health benefits, such as improving quality of life and mobility, and reducing disabilities. Conversely, lack of physical activity is considered a risk factor for increasing chronic diseases, functional dependence and mortality. Patients with low back pain (LBP) often report disability to perform daily activities. Also, decreased physical activity level can lead to low back pain. Objectives: The aim of the study was to determinewhether increasing the level of physical activity affects the low back pain or not in the population of univesity students.

Methods: The cross-sectional study included 350 students (181 females and 169 males) with a mean age of $19.8 \pm 1.9$ years. LBP was determined using the validated Oswestry Disability Index (ODI). ODI consist of ten items and are completed in reference to the patient's functional status "today". Physical activity level was evaluated by the short form of the International Physical Activity Questionnaire (IPAQ). IPAQ is a scale to be recorded at different levels of physical activity time in the last week. IPAQ is a scale to be recorded at different levels of physical activity time in the last week. Individuals whose score is lower than 600 MET are described as inactive (IPAQ 1), between 600-1500 MET (IPAQ 3) are described as minimal active and higher than 3000 MET (IPAQ 5)are described as active. For the statistical analyzing we used spearman correlation test.

Results: According to the short form of IPAQ, \%14.8 percent of students were found inactive, $\% 48.1$ percent of students were found minimal inactive and $\% 37.1$ percent of students were found active. The total score of Oswestry disability index was found $6,2 \pm 5,0$. There was a significant difference between the Oswestry disability index score of inactive, minimally active and active groups $(p=0,02)$. In addition to this, between the Oswestry disability index and total score of IPAQ was found a weak correlation in the negative direction $(p=0,01 ; r=-0,184)$. Between the IPAQwhich means vigorous intensity activity, and Oswestry disability index was found a weak correlation in the negative direction $(p=0,00 ; r=-0,190)$. And also between IPAQ3, which means modarate intensity activity, and Oswestry disability index was found a significant but weak correlation in the negative direction $(p=0,03 ; r=-0,157)$. Between Oswestry disability index and sedentary activity and walking was not found any significant correlation $(p>0.005)$.

Conclusions: According to our study, increasing the level of physical activity may reduce low back pain. Especially vigorous and modarate intensity activity can be effective in prevention from low back pain. For reducing of low back pain, sedentary activity and walking did not have any significant effect in our study. Therefore, the level of physical activity should be increased at the young age to prevent of low back pain which is a major health problem.

References:

[1] Saglam, Melda, et al. International physical activity questionnaire: reliability and validity of the Turkish version. Perceptual and motor skills 111.1 (2010): 278-284.

[2] Fairbank, Jeremy CT, and Paul B. Pynsent. The Oswestry disability index. Spine 25.22 (2000): 2940-2953.

Disclosure of Interest: None declared

DOI: 10.1136/annrheumdis-2017-eular.4953

\section{SAT0607 DISCOVERY OF A SMALL MOLECULE INHIBITOR OF THE WNT PATHWAY (SM04755) AS A POTENTIAL TOPICAL TREATMENT FOR TENDINOPATHY}

V. Deshmukh, T. Seo, M. Ibanez, J. Stewart, B. Hofilena, Y. Yazici. Samumed, LLC, San Diego, CA, United States

Background: Tendinopathy is an inflammatory and degenerative disorder caused by injuries or overuse. It can progress to a chronic condition with failed healing, tendon fibrosis and micro-tears that lead to pain and sometimes rupture. Current therapeutic options focus mainly on pain relief rather than treatment of underlying disease. The Wnt pathway is upregulated in tendinopathy and has an important role in inflammation, fibrosis and tenocyte differentiation.

Objectives: SM04755, a novel, topical Wnt pathway inhibitor, was evaluated in preclinical studies to determine its potential to inhibit inflammation, reduce fibrosis and increase tenocyte differentiation, thereby promoting tendon healing.

Methods: Anti-inflammatory activity was measured by TNF- $\alpha$ and IL- 6 secretion using ELISA in lipopolysaccharides (LPS) or anti-CD3/anti-CD28 stimulated peripheral blood mononuclear cells (PBMCs). Differentiation of human mesenchymal stem cells (hMSCs) and rat tendon derived stem cells (rTDSCs) into tenocytes was measured by high-content imaging for tenocyte markers scleraxis A (SCXA), tenomodulin and tenascin C. Pharmacokinetics were evaluated following topical application in rats. In vivo efficacy of SM04755 was evaluated in a single injection, collagenase-induced acute rodent tendinopathy model and a chronic, multiple injection, failed healing model, by scoring histological indicators of tendon health. Inflammation was measured by chemokine ligand 1 (CXCL1) levels in plasma by ELISA and pro-inflammatory markers (IL-6, TNF- $\alpha$, IL-1 $\beta$, IFN- $\gamma$, IL- 8 ) in the tendon by qPCR. Tendon regeneration and healing were evaluated by qPCR based gene expression of tenocyte differentiation markers SCXA, tenomodulin and tenascin C, Type I/Type III collagen ratio and polarized light microscopy using Sirus Red staining. Pain in the rodent model was evaluated by measuring weight distribution with an incapacitance meter.

Results: SM04755 potently inhibited cytokine secretion in LPS and antiCD3/anti-CD28 stimulated PBMCs $\left(E_{50}=500 \mathrm{nM}\right)$. SM04755 induced expression of tenocyte markers in differentiated hMSCs and rTDSCs $\left(E_{50}=200 \mathrm{nM}\right)$. A single topical application of SM04755 resulted in tendon concentrations $>\mathrm{EC}_{50}$ for up to $24 \mathrm{hrs}$, with minimal systemic exposure or toxicity. In both the acute and failed healing tendinopathy models, SM04755 $(10 \mathrm{mg} / \mathrm{ml})$ treatment improved tendon morphology (Figure A), significantly increased mean tendon health 